\title{
Use of lectin histochemistry in pancreatic cancer
}

\author{
C K CHING, R BLACK, T HELLIWELL, A SAVAGE, H BARR, J M RHODES
}

From the Departments of Medicine and Histopathology, The University of Liverpool, the Department of Surgery, Rayne Institute, University College, London, and the Department of Histopathology, Selly Oak Hospital, Birmingham

SUMMARY Lectin peroxidase histochemical analysis was carried out on pancreatic tissue from patients with pancreatic carcinoma and chronic pancreatitis and from subjects with normal pancreas to find a tumour specific pattern of lectin binding that would aid histological and cytological diagnosis. There were striking differences between the lectin binding characteristics of the different cell types in the normal pancreas. Acinar cells were uniformly positive for binding with wheat germ agglutinin and soy bean agglutinin while islet cells were usually negative for these lectins. Ulex europaeus I lectin however, was found not to be specific for endothelium, showing positivity also for acinar and ductal tissue. Griffonia simplicifolia II lectin was found to be highly specific for ductal epithelium, and because of this was tested in a hamster pancreatic cancer model where it was not specific for ductal epithelium, reflecting differing carbohydrate expression in the hamster pancreas. Pancreatic carcinomas and chronic pancreatitis bound all five lectins without any qualitative distinction from each other or from normal pancreatic tissue, but there was increased intensity of peanut agglutinin binding to secreted mucins in pancreatic carcinoma, which may be of potential use in radiolabelled lectin scanning.

Many tumours of epithelial origin secrete abnormal glycoproteins which may be useful as tumour markers for diagnosis or screening. Although these are commonly detected using monoclonal antibodies these oncodevelopmental antigens differ from normally expressed glycoproteins mainly in their carbohydrate side chain structure rather than in their protein core.'

Lectins are plant or animal proteins or glycoproteins with specificity for individual sugars or groups of sugars, which makes them powerful tools for detecting changes in the carbohydrate structure of glycoproteins. There are now many published reports which show that lectin histochemical analysis may identify malignant or pre-malignant cells. ${ }^{2-10}$ The distinction between malignant and normal tissue is manifest either by the expression of a tumour related glycoprotein which is usually of an immature (oncofetal) type, or the absence of a normally expressed glycoprotein on the cell surface. The lectin peanut agglutinin (PNA, galactose 1-3 NAc galactosamine binding) has proved particularly useful for detecting

Accepted for publication 20 October 1987 glycoprotein changes in epithelial tumours including colon, ${ }^{3}$ breast, ${ }^{4}$ and stomach. ${ }^{5}$

Because of the poor results of surgery in pancreatic cancer there is an increasing tendency to attempt preoperative tissue diagnosis. This is often achieved by cytological analysis of aspirated cells or pancreatic juice. Cytological analysis of the pancreas may be difficult to interpret, " so any histochemical technique which could distinguish neoplastic from non-neoplastic cells would be helpful. Such a technique would also help to distinguish between adenocarcinoma and the distorted glands of chronic pancreatitis which often causes a problem in the interpretation of small needle biopsy specimens. Specific changes in tissue or secreted glycoprotein structure, identifiable by lectin binding might also help to identify further tumour markers which could be used for the development of serological tests.

The normal pancreatic ductal epithelial surface has previously been shown to bind Lotus tetragonolobus A (L-fucose binding), wheat germ agglutinin, Ricinus communis A I (D-galactose binding), and Ulex europaeus I lectin ${ }^{12}$ (table 1). The latter three lectins bound to intracytoplasmic supranuclear regions which correspond to the secretory granules. In the 
Table 1 List of lectins used

\begin{tabular}{llll}
\hline Origin of Lectins & Abbreviation & Specificity & Inhibitory/binding sugar \\
\hline Arachis hypogaea (peanut) & PNA & $\beta$-D-Gal $(1 \rightarrow 3)$-D-GalNAC D Gal & D-Galactose \\
Ulex europaeus (Gorse) & UEA I & $\alpha$-L-Fucose & L-Fucose \\
Wheat germ & WGA & Sialic acid Glc. NAC & N-Acetylglucosamine \\
Soy bean & SBA & $x$-or- $\beta$-D-GalNAC $\alpha$-D-Gal & D-Galactose \\
Griffonia simplicifolia & GSII & Glc.NAC & N-Acetylglucosamine \\
\hline
\end{tabular}

same study ductal intraluminal secretions bound Helix pomatia (N-Acetyl galactosamine binding), wheat germ agglutinin, and Ulex europaeus I lectins. In another small study of peroxidase labelled lectin binding, in snap frozen pancreatic tissue obtained from six patients with pancreatic carcinoma, six with normal pancreas, and two with chronic pancreatitis, Ulex europaeus I lectin bound preferentially to the cell surface of carcinoma cells in contrast to the predominantly intracytoplasmic binding of the same lectin in normal and chronic pancreatic tissue. Although PNA bound faintly, if at all, to normal pancreas in this study, it failed to differentiate malignant from chronically inflamed pancreatic tissue. ${ }^{13}$

Lectin histochemical analysis of normal and $\mathrm{N}$ nitroso-bis-(2-oxopropyl)amine (BOP) induced pancreatic carcinoma in the hamster was performed by Pour using nine different lectins. ${ }^{14}$ No qualitative difference in lectin binding was found between normal and malignant hamster pancreas.

In previous studies we have found, ${ }^{10}$ like Kuhlmann, ${ }^{15}$ that lectin peroxidase histochemistry can be successfully performed on formalin fixed tissue, and this allowed us to perform a larger study on pancreatic tissue to clarify the lectin binding characteristics of the normal and diseased pancreas.

\section{Material and methods}

Samples of pancreatic tissue were obtained at laparotomy from patients with pancreatic carcinoma ( $\mathrm{n}=13$, six needle and seven wedge biopsies), chronic pancreatitis $(n=13$, four needle and nine wedge biopsies) and normal pancreas $(n=14$, eight needle and six wedge biopsies). Tissue was fixed in $10 \%$ formaldehyde. Pancreatic biopsy specimens from three normal and three chronic pancreatitic pancreases, as well as from three normal and three carcinomatous hamster pancreases were snap frozen in liquid nitrogen and stored at $-70^{\circ} \mathrm{C}$ until sectioned. Carcinoma had been induced in the hamsters using $\mathrm{N}$ nitroso-bis-(2-oxopropyl)amine (BOP) by the method of Pour et al. ${ }^{16}$ Horseradish peroxidase-labelled lectins were purchased from EY laboratories, USA. The lectins used and their binding characteristics are shown in table 1.

Routine histological analysis was performed with haematoxylin and eosin staining to identify the normal and abnormal areas for study. Lectin histochemical analysis was performed according to the method of Kuhlmann et al. ${ }^{15}$ Five $\mu$ m sections were mounted on acetone cleaned slides, dewaxed, and passed from absolute ethanol into phosphate buffered saline $(\mathrm{pH}$ $7 \cdot 2$ ). Five $\mu \mathrm{m}$ sections were also obtained in duplicates from the snap frozen human pancreatic specimens. One of each pair was left to thaw out for 16 hours at room temperature to assess the effect of autolysis on lectin binding. Syrian hamster pancreatic sections were processed similarly but autolysis was omitted. All sections were incubated in $1 \%$ hydrogen peroxide in PBS for one hour to abolish endogenous peroxidase activity, washed in PBS, and incubated with the appropriate peroxidase labelled lectin (concentration $0.002 \mathrm{mg}$ lectin conjugate $/ \mathrm{ml}$ ). Control experiments were performed in which the lectins were preincubated with the appropriate inhibitory sugars (concentration $0.2 \mathrm{M}$ ) for one hour (table 1). All sections were incubated for 24 hours at $4^{\circ} \mathrm{C}$ on a tilting deck. Unreacted conjugates were washed off with three changes of PBS and peroxidase activity shown by incubation with 3,3' diaminobenzidine and hydrogen peroxide. After further washing in PBS sections were counterstained with haematoxylin and mounted. The slides were viewed using a conventional microscope.

\section{Results}

\section{HUMAN PANCREAS}

Each section was scored as either positive or negative with regard to acini (surface, intracytoplasmic, canalicular), ducts (surface, intracytoplasmic), and intraductal mucus. The results are summarised in table 2. The control studies showed complete lack of staining following preincubation of lectin with inhibiting sugar, confirming the specificity of lectin binding.

The lectin binding characteristics of the snap frozen normal and chronic pancreatitic specimens resembled the formalin fixed pancreas specimens, but the deliberately autolysed pancreatic specimens were more strongly and diffusely stained by all five lectins.

In paraffin embedded sections Griffonia simplicifolia II (GSII) specifically stained ductal cells with no staining of acinar tissue (fig 1 ) in normal pancreas. Ten of 13 pancreatic carcinomas and eight of 13 cases 
Table 2 No of cases positively stained by each lectin in normal and diseased pancreas

\begin{tabular}{|c|c|c|c|c|c|c|c|c|c|}
\hline & \multicolumn{3}{|c|}{ Normal $(n=14)$} & \multicolumn{3}{|c|}{ Pancreatic carcinoma $(n=13)$} & \multicolumn{3}{|c|}{ Chronic pancreatitis $(n=13)$} \\
\hline & $\overline{A c i n i}$ & Duct & Mucus & $\overline{A c i n i}$ & Duct & Mucus & $\overline{A c i n i}$ & Duct & Mucus \\
\hline $\begin{array}{l}\text { PNA } \\
\text { UEA-1 } \\
\text { SBA } \\
\text { WGA } \\
\text { GSII }\end{array}$ & $\begin{array}{r}8 \\
14 \\
14 \\
14 \\
0\end{array}$ & $\begin{array}{r}8 \\
10 \\
14 \\
14 \\
9\end{array}$ & $\begin{array}{r}7 \\
10 \\
14 \\
14 \\
5\end{array}$ & & $\begin{array}{l}10 \\
13 \\
13 \\
13 \\
10\end{array}$ & $\begin{array}{r}9 \\
13 \\
13 \\
13 \\
5\end{array}$ & $\begin{array}{r}9 \\
12 \\
13 \\
13 \\
0\end{array}$ & $\begin{array}{r}10 \\
12 \\
13 \\
13 \\
8\end{array}$ & $\begin{array}{r}6 \\
12 \\
13 \\
13 \\
6\end{array}$ \\
\hline
\end{tabular}

of chronic pancreatitis also showed GSII binding to ductal cells and some areas of intraductal mucus. This varied from patchy to intense diffuse staining, but there was no distinction between GSII binding to neoplastic and non-neoplastic epithelium.

Peanut agglutinin (PNA) showed a disappointing lack of specificity for carcinoma. Eleven of 13 cases of pancreatic carcinoma, 11 of 13 of chronic pancreatitis, and nine of 14 of normal pancreas showed some degree of PNA positivity, which, in most cases, was general-

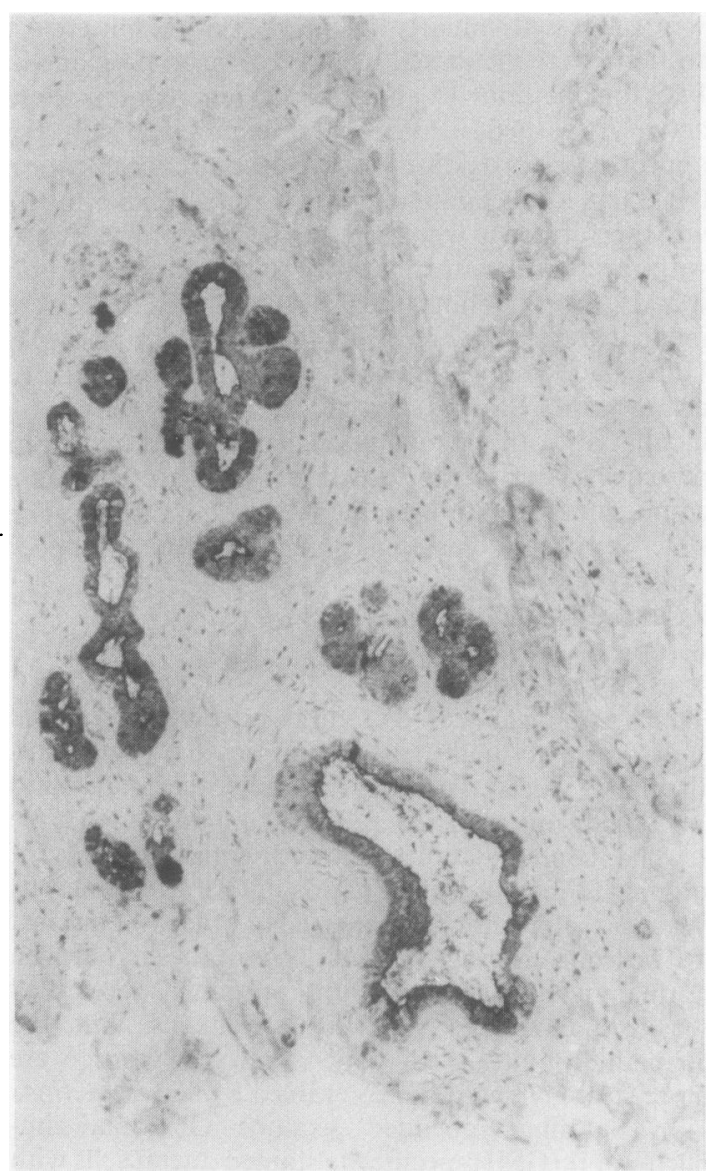

Fig 1 Normal pancreas showing GSII binding to ductal epithelium. (GSII peroxidase and haematoxylin). ised with binding to acinar or ductal tissue (surface and intracytoplasmic) or both. On an arbitary scale of $0-4$, however, the intensity of PNA binding to the intraductal mucus was $50 \%$ stronger in pancreatic carcinoma (mean grade of 1.8 , range $0-4$ ) than in chronic pancreatitis or normal pancreas (both with a mean grade of 1.2 and range $0-4$ ). Furthermore, four of the 11 PNA positive pancreatic cancers showed no staining of adjacent normal pancreatic tissues (figs 2 and 3).

Nearly all the cases in each group showed diffuse UEA-I binding to acinar cells, ductal cells, and intraductal mucus. UEA-I was bound to the cell surface and cytoplasm in normal and chronically inflamed pancreas, as well as in pancreatic cancer.

Soy bean agglutinin (SBA) and wheat germ agglutinin (WGA) showed no differential binding between neoplastic and non-neoplastic pancreatic tissue. All sections were strongly stained with binding of SBA and WGA to the cell surface, the cytoplasm of acinar and ductal cells, and the intraductal mucus. Islet cells were only occasionally stained by these two lectins (nine of 40 by WGA and three of 40 by SBA), as well as by UEA-I (five of 40), but were consistently negative for PNA (0 of 40).

All the lectins studied bound to connective tissue. Vascular endothelium was shown to bind PNA, SBA, and WGA in addition to UEA-I.

\section{SYRIAN HAMSTER PANCREAS}

Normal and malignant Syrian hamster pancreases showed intense diffuse staining by all five lectins used and, unlike the human pancreas, failed to show selective GS II lectin affinity for ductal tissue.

\section{Discussion}

PNA showed a disappointing lack of qualitative specificity for neoplastic pancreatic tissue, although it tended to bind more extensively and intensely than in normal tissue, particularly to mucin contained within the tumour. This difference is difficult to quantify histochemically but may reflect increased production of incompletely glycosylated, immature glycoproteins as a result of malignant transformation. This quantitative difference might be sufficient to merit a trial of 


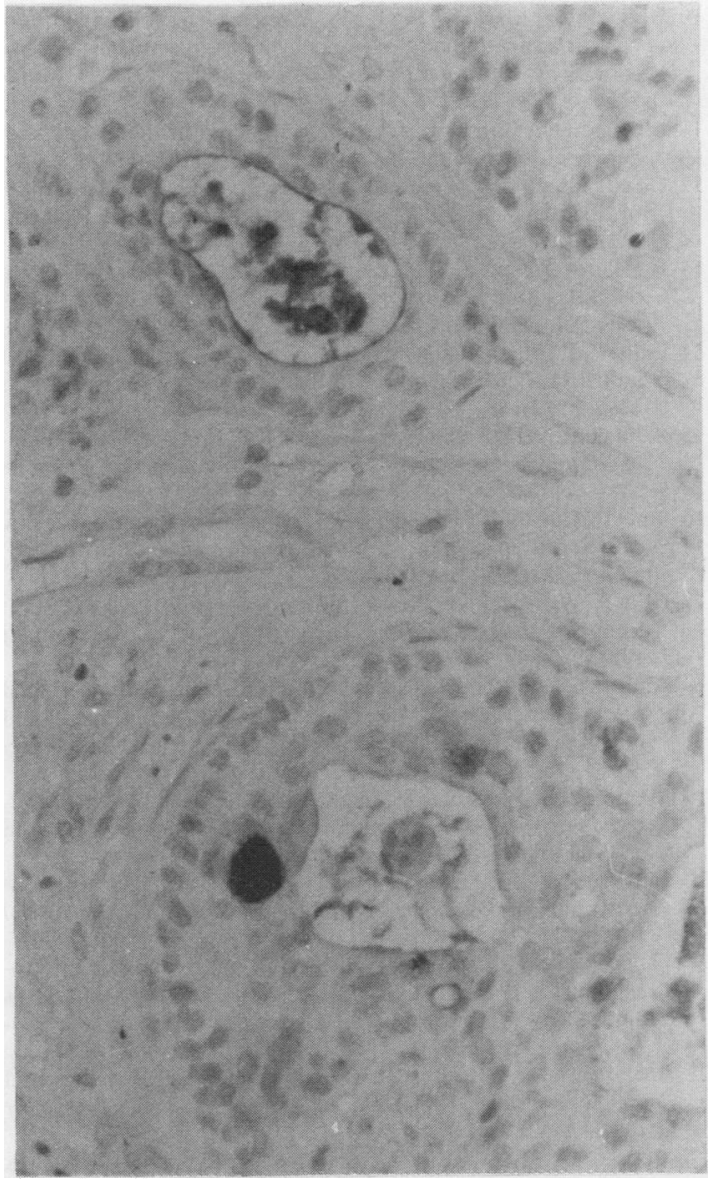

Fig 2 Pancreatic carcinoma showing PNA binding to intraductal mucus and ductal surface epithelium. (PNA peroxidase and haematoxylin).

radiolabelled PNA scanning in pancreatic cancer as it has been shown that this can be safely performed in man. ${ }^{17} 18$ The lack of qualitative specificity results from the non-specific binding of PNA to normal and inflamed pancreatic tissue. This is unlike the case in colon and breast where there is good differentiation between normal and neoplastic tissue by this lectin. ${ }^{34}$

Our findings differ from those of Raedler et al,${ }^{13}$ who found that PNA bound faintly, if at all, to normal pancreas and weakly to chronic pancreatitic tissue while binding more strongly to carcinoma. Furthermore, in our study UEA-I bound to all elements of pancreatic tissue whether neoplastic or non-neoplastic, and there was no predilection for neoplastic cell surfaces, which was noticed by Raedler et al. ${ }^{13}$ The difference in results between Raedler and ours are difficult to account for. They are unlikely to be due to

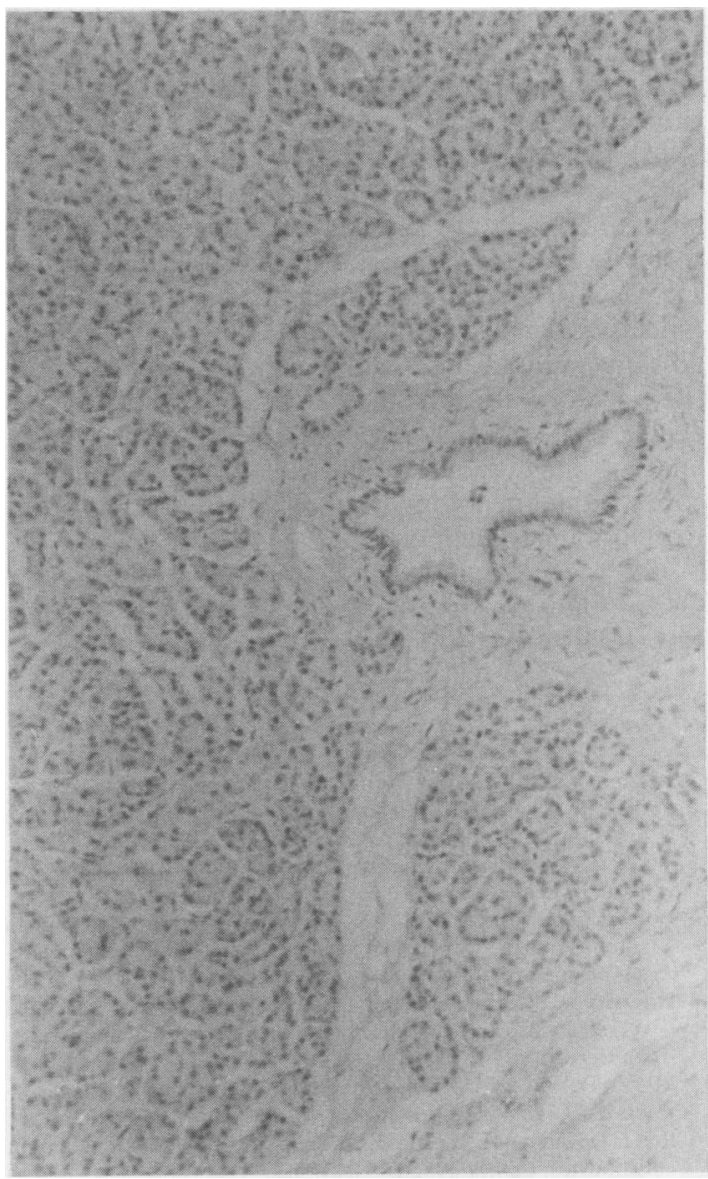

Fig 3 Normal pancreas adjacent to carcinoma showing lack of PNA binding (PNA peroxidase and haematoxylin).

differences in the technique of specimen collection as we found similar results whether using snap frozen or formalin fixed pancreatic tissue.

This study confirms that UEA-I is not a specific marker for vascular endothelium, as has been suggested, ${ }^{19}$ as it binds to normal and neoplastic pancreatic tissue. It has also been shown to bind malignant colonic epithelium. ${ }^{10}$

While most human pancreatic carcinomas are thought to be of ductal origin, ${ }^{20}$ there has been considerable debate about the cell of origin of the chemically induced hamster pancreatic cancer model. It has been varyingly reported to be derived from acinar cells ${ }^{21}{ }^{22}$ or from ductal cells. ${ }^{2324}$ GSII was highly specific for human pancreatic ductal tissue in this study. We therefore extended the study to investigate its use in the Syrian hamster pancreas, but it was 
shown to be disappointingly non-specific in the hamster pancreas and is therefore unlikely to help in differentiating the cell of origin in this experimental cancer model.

In conclusion, lectin histochemical analysis failed to show any qualitative abnormalities in glycoprotein structure which are specific for pancreatic cancer in formalin fixed, paraffin embedded tissues. There was increased PNA lectin binding, however, to secreted mucins in pancreatic cancer, and this may be exploitable by radiolabelled lectin scanning. Furthermore, there are striking differences in lectin binding between acinar, islet, and ductal cells, and lectin histochemical analysis may therefore prove a useful adjunct to conventional histology in cases where it is important to determine the cell type.

CKC is an Amelie Waring research fellow of the British Digestive Foundation.

\section{References}

1 Feizi T. Demonstration by monoclonal antibodies that carbohydrate structures of glycoproteins and glycolipids are oncodevelopmental antigens. Nature 1985;314:53-7.

2 Walker R. The use of lectins in histopathology. Histopathology 1985;9:1121-4.

3 Boland CR, Montgomery CK, Kim YS. Alterations in human colonic mucin occurring with cellular differentiation and malignant transformation. Proc Natl Acad Sci USA 1982;79:2051-5.

4 Howard DR, Ferguson P, Batsakis JG. Carcinoma associated cytostructural antigenic alteration. Detection by lectin binding. Cancer 1981;47:2872-7.

5 Martin B, Wilbur AF. Lectin binding to human gastric adenocarcinomas and adjacent tissues. Am J Pathol 1985;119:279-87.

6 Ree HJ, Kadin ME. Lectin distinction of benign from malignant histocytes. Cancer 1985;56:2046-50.

7 Veerman AJP, Hogeman PHG, Huismans DR, Van Zantwijk CH, Bezemer PD. Peanut agglutinin, a marker for T-cell Acute Lymphoblastic Leukaemia with a good prognosis. Cancer Res 1985:45:1890-3.

8 Dabelsteen E, Mackenzie ICT. Expression of Ricinus communis receptors on epithelial cells in oral carcinomas and oral wounds. Cancer Res 1978;38:4676-80.

9 Prime SS, Rosser TJ, Malamos D, Shepherd JP, Scully C. The use of the lectin ulex europaeus to study epithelial cell differentiation in neoplastic and non-neoplastic oral white lesions. J Pathol 1985;147:173-9.
10 Rhodes JM, Black R, Savage A. Glycoprotein abnormalities in colonic carcinomata, adenomata and hyperplastic polyps shown by lectin peroxidase histochemistry. J Clin Pathol 1986;39:1331-4.

11 Mitchell ML, Carney CN. Cytologic criteria for the diagnosis of pancreatic carcinoma. Am J Clin Pathol 1985;83:171-6.

12 Geleff S, Bock P. Pancreatic duct glands II: lectin binding affinities of ductular epithelium, ductular glands and Brunner glands. Histochemistry 1984;80:31-8.

13 Raedler A, Schmiegel WH, Raedler E, Arndt R, Thiele HG. Lectin defined cell surface glyconjugates of pancreatic cancer cells and their non-malignant counterparts. Exp Cell Biol 1983;51:19-28.

14 Pour PM, Burnett D, Uchida E. Lectin binding affinities of induced pancreatic lesions in the hamster model. Carcinogenesis 1985;6:1775-80.

15 Kuhlmann WD, Peschke P, Wurster K. Lectin-peroxidase conjugates in histopathology of gastrointestinal mucosa. Virchows Arch (Pathol Anat) 1983;398:318-28.

16 Pour PM, Althoff J, Krüger FW, Mohr U. A potent pancreatic carcinogen in Syrian hamsters: $\mathrm{N}$-nitrosobis (2-oxopropyl) amine. JNCI 1977;58:1449-53.

17 Holt S, Wilkinson A, Suresh MR, et al. Radiolabelled peanut lectin for the scintigraphic detection of cancer. Cancer Lett 1984;25:55-60.

18 Abdi EA, Kamitomo VJ, McPherson TA, et al. Radioiodinated peanut lectin: clinical use as a tumour-imaging agent and potential use in assessing renal-tubular function. Eur $\mathrm{J} \mathrm{Nucl}$ Med 1986;11:350-4.

19 Meittinen M, Holthofer H, Lehto V-P, Meittinen A, Virtanen I. Ulex europaeus I lectin as a marker for tumours derived from endothelial cells. Am J Clin Pathol 1983;79:32-6.

20 Cubilla AL, Fitzgerald PJ. Cancer of the pancreas (nonendocrine): a suggested morphologic classification. Semin Oncol 1979;6:285.

21 Flaks B, Moore MA, Flaks A. Ultrastructure analysis of pancreatic carcinogesis VI. Early changes in hamster acinar cells induced by $\mathrm{N}$-nitroso-bis (2-hydroxypropyl) amine. Carcinogenesis 1982;3:1063-70.

22 Bockman DE. Cells of origin of pancreatic cancer. Experimental animal tumours related to human pancreas. Cancer 1981;47:1528-34.

23 Moore MA, Takahashi M, Ito N, Bannasch P. Early lesions during pancreatic carcinogenesis induced in syrian hamster by DHPN or DOPN I. Histologic, histochemical and radioautographic findings. Carcinogenesis 1983;4:431-7.

24 Moore MA, Takahashi M, Ito N, Bannasch P. Early lesions during pancreatic carcinogenesis induced in Syrian hamster by DHPN or DOPN II. Ultrastructural findings. Carcinogenesis 1983;4:439-48.

Requests for reprints to: Dr C K Ching, Department of Medicine, The University of Liverpool, PO Box 147, Liverpool L69 3BX, England. 